\title{
The Prehistory of Antibiotic Resistance
}

\author{
Julie Perry, Nicholas Waglechner, and Gerard Wright \\ M.G. DeGroote Institute for Infectious Disease Research, Department of Biochemistry and Biomedical \\ Sciences, DeGroote School of Medicine, McMaster University, Hamilton, Ontario L8S 4K1, Canada \\ Correspondence: wrightge@mcmaster.ca
}

Antibiotic resistance is a global problem that is reaching crisis levels. The global collection of resistance genes in clinical and environmental samples is the antibiotic "resistome," and is subject to the selective pressure of human activity. The origin of many modern resistance genes in pathogens is likely environmental bacteria, including antibiotic producing organisms that have existed for millennia. Recent work has uncovered resistance in ancient permafrost, isolated caves, and in human specimens preserved for hundreds of years. Together with bioinformatic analyses on modern-day sequences, these studies predict an ancient origin of resistance that long precedes the use of antibiotics in the clinic. Understanding the history of antibiotic resistance is important in predicting its future evolution.

$T_{\mathrm{r}}^{\mathrm{h}}$ he emergence of antibiotic-resistant human pathogens at a rate that exceeds new drug discovery threatens to end an age of unparalleled achievement in modern medicine. Beyond the management of infectious disease, advances in organ transplantation, orthopedic surgery, renal dialysis, and cancer chemotherapy all depend on our ability to control infection. The Center for Disease Control and Prevention (CDC) have declared 15 antibiotic-resistant human pathogens as "urgent" or "serious" threats to human health, and estimate that two million people are sickened every year in the United States alone by antibiotic-resistant infections (see www.cdc.gov/drugresistance/ biggest_threats.html). Antibiotics have only been in clinical use for $\sim 80$ years, yet we face the imminent possibility that they will be lost as therapeutics as a result of resistance. How did we get here so quickly? Were pathogens always antibiotic-resistant? If not, how did resistance develop? This review will explore the ancient nature of antibiotic resistance in the context of the concept of the antibiotic "resistome."

\section{THE ANTIBIOTIC “RESISTOME"}

The origins of clinical antibiotic resistance can be found in the environment. Most antibiotics in medical or agricultural use are derived from or produced by a group of soil-dwelling bacteria called the Actinomycetes (the most notable genus for antibiotic production being Streptomyces). These organisms are prolific producers of specialized metabolites (so-called "natural products"), including the antibiotics streptomycin, tetracycline, chloramphenicol, erythromycin, and vancomycin. Of course, these organisms must also be resistant to the antibiotics they produce, or they would succumb to their

Editors: Lynn L. Silver and Karen Bush

Additional Perspectives on Antibiotics and Antibiotic Resistance available at www.perspectivesinmedicine.org

Copyright (C) 2016 Cold Spring Harbor Laboratory Press; all rights reserved; doi: 10.1101/cshperspect.a025197 Cite this article as Cold Spring Harb Perspect Med 2016;6:a025197 
J. Perry et al.

own toxic metabolites. In fact, antibiotic producers may have been the original sources for many of the antibiotic-resistance genes circulating in the clinic today (Benveniste and Davies 1973; Humeniuk et al. 2002). However, most Streptomyces are resistant to an average of seven to eight antibiotics, including newly developed and clinically important therapeutics (D'Costa et al. 2006). How did these environmental organisms become multidrug-resistant? Bacteria are unique in that they acquire genes from the parent microorganism during division (vertical gene transfer), but can also acquire genes from the community at large (horizontal gene transfer, first shown for aminoglycoside resistance [Benveniste and Davies 1973], and reviewed in Ochman et al. 2000; van Elsas and Bailey 2002; Aminov and Mackie 2007; Aminov 2011; Skippington and Ragan 2011; Stokes and Gillings 2011; Wiedenbeck and Cohan 2011). It is now clear that the environment is a vast source of new and emerging resistance genes (Wright 2010) accessible by members of the microbial community via horizontal gene transfer. The global collective of all resistance genes in the clinic, in the microbiome of humans and animals, and in environmental bacteria is the antibiotic "resistome." The diversity and extent of the resistome across both environmental and human microbiomes suggests a long evolutionary history, which has been explored in the recent studies discussed below.

\section{Links to the Environment and Gene Mobilization}

The permafrost found in the Canadian High North offers a genomic glimpse into the past, as its permanently frozen nature has preserved DNA for tens to hundreds of thousands of years. In a defining study, D'Costa et al. (2011) showed the presence of gene-encoding resistance to $\beta$ lactam, tetracycline, and glycopeptide antibiotics in metagenome samples of 30,000-year-old permafrost, which also contained DNA belonging to woolly mammoths and other animal and plant species unique to the Pleistocene. Detailed analysis of the vancomycin resistance gene cluster in this metagenome revealed conservation of gene sequence and synteny with modern resistance clusters in the clinic as well as protein function and structure. Other studies have shown the presence of resistance genes in Siberian permafrost (Petrova et al. 2009) and showed working resistance genes from at least 5000 years ago using functional metagenomics (Perron et al. 2015). Isolated instances of resistance genes in human commensals and pathogens have also been documented. The gut microbiome of a pre-Columbian Andean mummy from Cuzco, Peru $\left({ }^{14} \mathrm{C}\right.$ dating of $980-1170$ AD) was recently found to harbor genes with homology to $\beta$-lactam, fosfomycin, chloramphenicol, aminoglycoside, macrolide, sulfa, quinolones, tetracycline, and vancomycin resistance genes (Santiago-Rodriguez et al. 2015). Antibiotic resistance elements were detected in the oral microbiome of four adult human skeletons from a medieval monastery (ca. 950-200 CE). Native resistance to aminoglycosides, $\beta$-lactams, bacitracin, bacteriocins, and macrolides and a near-complete plasmid-encoded conjugative transposon carrying efflux pump genes with high homology to CTn5 of Clostridium difficile were found in these samples, implying that the human microbiome has long served as a reservoir of resistance genes accessible to pathogens even in the absence of the strong selective pressure of modern antibiotic availability (Warinner et al. 2014). The first bacterial isolate deposited in the United Kingdom's National Collection of Type Cultures (NCTC) was a strain of the dysentery-causing bacterium Shigella flexneri that killed a young soldier in World War I. Despite being isolated in 1915 (long before the discovery and use of antibiotics), this human pathogen was found to carry resistance genes for penicillin and erythromycin when it was revived and sequenced in 2014 (Mather et al. 2014).

These instances of resistance, spanning roughly 30,000 years, establish that the resistome is indeed ancient. We know, however, that resistance in the environment has increased significantly because of the introduction of antibiotics in agriculture and in medicine (Knapp et al. 2010), and that sensitivity has been steadily replaced with resistance in the clinic (Chait et al. 2012). There is no question that the pro- 
duction and use of antibiotics in modern times has influenced the dissemination of resistance, and it is now a matter of staying one step ahead of the microbes. Although research has traditionally focused on the present-day clinical resistome, we have just begun to probe the vastness of the environmental and ancient resistomes. Understanding how resistance develops over time and moves among microorganisms will be of immense value in predicting the future course of resistance development. The remainder of this article will explore the ancient resistome in greater detail.

\section{HOW OLD IS RESISTANCE PREDICTED TO BE?}

Assigning an evolutionary "age" to a gene is no small feat, and requires the comparison of genes from different species that have evolved from a common ancestral gene ("orthologs") (Tatusov et al. 1997). Divergence times can be determined from the appearance of major transitions in the fossil record, and used to calibrate the expected level of identity among sets of conserved orthologs across major groups in the tree of life (Landan et al. 1990; Weigel et al. 1988). The result uses the Grishin equation $q$ $=\ln (1+2 D) / 2 D($ Grishin 1995), an expression that relates the average fraction of unchanged residues $q$ to a distance $D$, which can be converted to a calibrated time of the last common ancestor (LCA) of the compared sequences. This is a nonlinear relationship between $q$ and $D$, and a linear relationship $D$ to time $(0.088 / t$ in millions of years) (Feng et al. 1997). These types of analyses require full genome sequence data from multiple organisms, and sophisticated informatics tools capable of sifting through large amounts of data. First attempts at dating resistance were made using traditional Sanger DNA sequencing on a limited number of biosynthetic genes (discussed below) (Landan et al. 1990). Although effective, these approaches offer a limited scope of analysis, because some prior knowledge of sequence is required to retrieve orthologs from multiple organisms. Advances in next-generation sequencing technologies and bioinformatics on the other hand are mak- ing unprecedented amounts of information available and generate data without prior sequence knowledge. As a result, analyses based on next-generation sequencing data are less subject to the sample bias inherent to Sanger sequencing-based methods. Gene dating based on both methods is discussed in more detail in the following sections.

\section{PREDICTIONS OF AGE BASED ON BIOSYNTHETIC CLUSTERS: TRADITIONAL SEQUENCING}

For producers of "natural products" with antibiotic activity, resistance must be as old as biosynthesis. Dating biosynthetic gene clusters can, therefore, give us a reasonable estimate of the age of resistance. In one of the first studies attempting to date antibiotic biosynthesis, cloning and traditional sequencing methods were used to date the transfer of isopenicillin- $N$ synthase (IPNS, one of the enzymes involved in the synthesis of the antibiotic penicillin) between a bacterium and three fungi (Weigel et al. 1988). The analysis included IPNS sequences from Penicillium chrysogenum, Aspergillus nidulans, and Cephalosporium acremonium, and the Grampositive bacterium Streptomyces lipmanii, and was performed assuming a constant rate of $10^{-9}$ nucleotide changes per site per year, to arrive at an estimated 370 million years, since gene transfer from bacteria to fungi. With the later discovery of a new IPNS in Flavobacterium, a Gram-negative bacterial genus, the hypothesis was revised to require a single horizontal transfer from bacteria to fungi before the Gram-positive/-negative split (Landan et al. 1990). This date estimation depends on the accuracy of the predicted rate of change of these sequences, which is different for prokaryotes and eukaryotes and likely has not remained constant because the sequences diverged (Lynch 2010).

Although Landan et al. (1990) focused their analysis on a single biosynthetic enzyme, Richard Baltz (2005) asked more broadly whether biosynthesis to specific antibiotics could be dated. Baltz (2005) reasoned that older antibiotics would be encountered more frequently than younger antibiotics in a drug discovery pro- 
J. Perry et al.

gram, because of the spread of biosynthetic genes vertically and horizontally in a population given evolutionary time. Baltz (2005) calculated that the average similarity among nine orthologs involved in streptomycin biosynthesis was $72.6 \%$, resulting in an estimate of $610 \pm 71$ million years since their LCA. Erythromycin, another commonly encountered antibiotic, was calculated to be $880 \pm 134$ million years old, using 18 sequences with an average of $62.9 \%$ identity. Similarly, six orthologous sequences involved in the production of the glycopeptide natural products balhimycin and chloroeremomycin shared on average $88 \%$ identity, resulting in an estimate of $240 \pm 12$ million years since their LCA. Baltz noted that the age of glycopeptide antibiotics may be less than his estimate, as the producing organism is rare and likely encountered less frequently in the soil than members of Streptomyces. Last, Baltz analyzed the biosynthetic genes of two organisms producing daptomycin, a newly discovered lipopeptide antibiotic. He calculated an LCA of $30 \pm 0.2$ million years based on an identity of $98.7 \%$ between two producing organisms. The 10 -fold difference in the predicted age of the rarely encountered daptomycin biosynthetic cluster versus the more frequently encountered antibiotics streptomycin and erythromycin supports the hypothesis that older antibiotics are frequently "rediscovered," because their biosynthetic clusters have had evolutionary time to disperse more widely in the metagenome (Baltz 2005). However, this analysis is based on detection of production under laboratory conditions. As more actinomycete genome sequences become available, we are increasingly recognizing that these strains harbor "silent" biosynthetic clusters and that laboratory production estimates of the frequency of production likely significantly underestimates the actual genetic diversity of biosynthesis.

The above-mentioned studies examine antibiotic biosynthesis, which is only an approximation of the age of resistance. Can resistance genes themselves been dated? In a series of papers, Barlow, Hall, and Salipante examined the origin and evolution of $\beta$-lactamases, the major resistance determinants of $\beta$-lactam antibiotics (Barlow and Hall 2002a,b, 2003a,b; Hall et al.
2003, 2004; Hall and Barlow 2004). The investigators proposed that the origin of the AmpC $\beta$-lactamase was chromosomal, because the phylogeny of chromosomal AmpC sequences matches the species phylogeny of the hosts. AmpC has presumably undergone multiple mobilizations to plasmids and to other species in the course of evolution (Barlow and Hall 2002a). A separate phylogenetic analysis of the OXA $\beta$-lactamase family also argues for multiple mobilizations to plasmids for this gene ( $\sim 42 \pm 9$ and $116 \pm 25$ million years ago), and a transfer of an OXA gene between Streptococcus and Bacillus between 320 and 520 million years ago (Barlow and Hall 2002a). The relative dates of three different classes of serine $\beta$-lactamases were also determined by Hall and Barlow (2003) using a structural alignment based on pairwise root mean square (RMS) distances. Structural distance was used rather than a sequence alignment because of the lack of detectable homology to these sequences. To root this phylogeny, a DD-peptidase structure from Streptomyces was used along with the EstB esterase from a species of Burkholderia. According to this analysis, the class $C \beta$-lactamases were earliest to diverge, followed by class $\mathrm{D}$. Both were outgroups compared with the class A $\beta$-lactamases, but because the RMS distances are not calibrated to a clock there is no way to provide dates for these splits (Hall and Barlow 2003). Rounding out the analysis of the $\beta$-lactamases, the subgroups of the class B metallo- $\beta$-lactamases were proposed to have two independent origins (Hall et al. 2004). Their tree of the B1+B2 subclass metallo- $\beta$-lactamases suggest that these sequences arose around one billion years ago, around the time the gammaproteobacteria diverged from the $\beta$-proteobacteria. The B3 subclass was proposed to date to 2.2 billion years ago, before the split of Gram-positive and Gram-negative Eubacteria.

\section{The Impact of Technology on Our Ability to Predict the History of Antibiotics}

Estimates of age based on a few sequences (like daptomycin, above) are less accurate than analyses generated with more sequencing data. This 
is an ideal time to revisit these estimates, as the genome sequencing of the class Actinomycetes has expanded dramatically with the advent of next-generation sequencing (1055 genome sequences listed at the National Center for Biotechnology Information [NCBI], along with further 7020 genome-sequencing projects as of December 2015, see ncbi.nlm.nih.gov/ Taxonomy/Browser/wwwtax.cgi). However, the development of new methods that make use of the information contained in whole genomes in general, instead of just handfuls of identifiable orthologs, is of utmost importance. To that end, novel techniques like single-cell sequencing and metagenomic analyses (the analysis of all DNA isolated directly from an environment without prior culture) are improving access to branches on the tree of life that have been previously undersampled (Jansson and Tas 2014). A recent series of analyses by the Dantas laboratory using culture-independent techniques have yielded an enormous wealth of resistance gene sequence data that will help in the phylogenetics and dating of resistance (Forsberg et al.2012,2014; Moore et al.2013; Pehrsson et al.2013; Gibson et al.2015). These studies have used "functional metagenomics," in which the entire microbial metagenome is cloned into an expression vector, transformed into an expression host (Escherichia coli) and selected on an antibiotic of interest. This technique captures novel resistance genes that would have otherwise been missed by traditional culture- or polymerase chain reaction (PCR)-based methods. Forsberg et al. (2012) first showed the power of this technique to link genes in the environment and the clinic, and offer evidence of horizontal transfer of resistance between these two environments. Increasing the number and diversity of sequences we can retrieve from an environment will lead to a better understanding of the evolutionary trajectory of current (and future) resistance elements.

Advances in sequencing go hand in hand with the software required for the detection and analysis of biosynthetic gene clusters (Blin et al. 2013; Cimermancic et al. 2014; Doroghazi et al. 2014) enabling finer analysis of this genome data in the identification of new clusters.
New proposals for the annotation and classification of biosynthetic gene clusters will provide the foundation to examine and reconstruct their evolutionary histories with unprecedented detail (Medema et al. 2015). As more gene sequences become available, phylogenies will improve accordingly; dating resistance determinants is often an accidental by-product of phylogenetic studies aimed at classifying newly discovered resistance determinants. The next section discusses the methodology of phylogenetics in greater detail.

\section{The Phylogenetics of Ancient Resistance}

It is possible to tie some of the evolutionary history of antibiotic resistance determinants to major evolutionary events inferred by other phylogeneticists, such as divergence of major lineages or the emergence of large groups of organisms (Battistuzzi and Hedges 2009). However, evolution is rarely linear, and gene gain, duplication, loss, and horizontal gene transfer in bacteria confound dating efforts with even the most sophisticated methods. Modern phylogenomic approaches have three goals: (1) inferring more accurate gene trees give a known species tree, (2) inferring a more accurate species tree given gene trees, and (3) joint estimation of both a species and gene tree (Boussau et al. 2013; Chan and Ragan 2013). The evolution of biosynthetic gene clusters should not be expected to occur in the same manner as independent sequences, and the method used to infer the phylogeny of whole clusters should be sensitive to the gain, loss, duplication, and innovation of new components. An excellent review by Aminov and Mackie (2007) provides further detail on the phylogenies of the tet genes (conferring tetracycline-resistance), the erm family of methyltransferases (conferring resistance to erythromycin), and the vanHAX cluster (encoding glycopeptide resistance genes).

\section{EXPERIMENTAL EVIDENCE FOR OLD RESISTANCE}

In silico analyses of orthologous gene sequences have predictive value, but is there experimental 
J. Perry et al.

evidence that resistance pre-dates the clinical use of antibiotics? Besides the studies mentioned in the introduction to this review (Mather et al. 2014; Warinner et al. 2014), few instances of resistance have been found in the limited number of sequenced of ancient microorganisms from human samples. The genome sequence of Vibrio cholera from 19th century Philadelphia did not reveal any candidate resistance genes apart from efflux (Devault et al. 2014), nor were any specific resistance genes found in several strains of the plague-causing bacillus Yersinia pestis isolated from the plague of Justinian (541-543 AD) (Wagner et al. 2014). It is not altogether surprising that resistance genes would be found sparingly in the human microbiome before the clinical use of antibiotics, because there would have been no selective pressure to maintain them. In contrast, environmental microorganisms have coevolved with antibiotics produced in Actinobacteria, and are more likely to harbor examples of resistance genes from long ago. An unparalleled source of ancient DNA is the permanently frozen soil known as "permafrost," found under an estimated 25\% of the earth's surface (Jansson and Tas 2014). Permafrost is defined as soil that has remained frozen for at least two consecutive years, but some Arctic and Antarctic permafrost has been frozen for 1-3 million years (Wagner et al. 2014). The DNA from permafrost can be isolated and queried experimentally for antibiotic resistance genes (among other things). The seminal work of D'Costa et al. (2011) on Beringian permafrost is complemented by studies showing that functional resistance genes can be retrieved from 5000-year-old DNA (Perron et al. 2015), and that resistance had mobilized to plasmids and transposons in ancient times (Mindlin et al. 2005; Petrova et al. 2011, 2014). Modern day microorganisms found in a cave that has been isolated from the surface for four million years have also been shown to harbor functional antibiotic resistance genes (Bhullar et al. 2012). A phylogenetic tree of macrolide phosphostransferases was generated using a sequence found in the genome of a cave organism (identified as Brachybacterium paraconglomeratum), and compared with a phylogeny of macrolide phosphotransferases from a terrestrial species of Brachybacterium (B. faecium DSM 4810) and environmental Bacillus cereus (Wang et al. 2015). Analysis of $10 \mathrm{~kb}$ upstream of and downstream from the $m p h$ revealed that MPHs from Brachybacterium strains from both cave and terrestrial origin cluster together as a separate group among known MPHs (Bhullar et al. 2012). The results of these studies provide direct experimental evidence that antibiotic resistance is ancient, and provide a glimpse into the evolutionary history of a natural environmental phenomenon.

\section{CONCLUSIONS}

Using both in silico methods and direct experimental analysis, we are beginning to unravel the complex natural history of antibiotic resistance. The importance of studying ancient resistance is twofold. First, cataloging the vast environmental reservoir of resistance genes provides advance notice as to what forms of resistance have the potential to emerge in the clinic under selective pressure. Second, understanding evolution has predictive value for resistance genes that are currently emerging as clinically important. Winston Churchill said "the farther backward you can look, the farther forward you are likely to see." This is especially true for antibiotic resistance where the past predicts and informs the future.

\section{ACKNOWLEDGMENTS}

This work in G.W.'s laboratory is funded by the Canadian Institutes of Health Research (CIHR) and by a Canada Research Chair in Biochemistry (to G.W.). N.W. is the recipient of a CIHR Fellowship.

\section{REFERENCES}

Aminov RI. 2011. Horizontal gene exchange in environmental microbiota. Front Microbiol 2: 158.

Aminov RI, Mackie RI. 2007. Evolution and ecology of antibiotic resistance genes. FEMS Microbiol Lett 271: 147- 161 .

Baltz RH. 2005. Antibiotic discovery from actinomycetes: Will a renaissance follow the decline and fall? SIM News 55: $186-196$. 
Barlow M, Hall BG. 2002a. Phylogenetic analysis shows that the OXA $\beta$-lactamase genes have been on plasmids for millions of years. J Mol Evol 55: 314-321.

Barlow M, Hall BG. 2002b. Origin and evolution of the AmpC $\beta$-lactamases of Citrobacter freundii. Antimicrob Agents Chemother 46: 1190-1198.

Barlow M, Hall BG. 2003a. Experimental prediction of the natural evolution of antibiotic resistance. Genetics 163: 1237-1241.

Barlow M, Hall BG. 2003b. Experimental prediction of the evolution of cefepime resistance from the CMY-2 AmpC B-lactamase. Genetics 164: 23-29.

Battistuzzi FU, Hedges SB. 2009. A major clade of prokaryotes with ancient adaptations to life on land. Mol Biol Evol 26: 335-343.

Benveniste R, Davies J. 1973. Aminoglycoside antibioticinactivating enzymes in actinomycetes similar to those present in clinical isolates of antibiotic-resistant bacteria. Proc Natl Acad Sci 70: 2276-2280.

Bhullar K, Waglechner N, Pawlowski A, Koteva K, Banks ED, Johnston MD, Barton HA, Wright GD. 2012. Antibiotic resistance is prevalent in an isolated cave microbiome. PLoS ONE 7: e34953.

Blin K, Medema MH, Kazempour D, Fischbach MA, Breitling R, Takano E, Weber T. 2013. antiSMASH 2.0-A versatile platform for genome mining of secondary metabolite producers. Nucleic Acids Res 41: W204-W212.

Boussau B, Szollosi GJ, Duret L, Gouy M, Tannier E, Daubin V. 2013. Genome-scale coestimation of species and gene trees. Genome Res 23: 323-330.

Chait R, Vetsigian K, Kishony R. 2012. What counters antibiotic resistance in nature? Nat Chem Biol 8: 2-5.

Chan CX, Ragan MA. 2013. Next-generation phylogenomics. Biol Direct 8: 3.

Cimermancic P, Medema MH, Claesen J, Kurita K, Wieland Brown LC, Mavrommatis K, Pati A, Godfrey PA, Koehrsen M, Clardy J, et al. 2014. Insights into secondary metabolism from a global analysis of prokaryotic biosynthetic gene clusters. Cell 158: 412-421.

D’Costa VM, McGrann KM, Hughes DW, Wright GD. 2006. Sampling the antibiotic resistome. Science 311: 374-377.

D'Costa VM, King CE, Kalan L, Morar M, Sung WW, Schwarz C, Froese D, Zazula G, Calmels F, Debruyne R, et al. 2011. Antibiotic resistance is ancient. Nature 477: 457-461.

Devault AM, Golding GB, Waglechner N, Enk JM, Kuch M, Tien JH, Shi M, Fisman DN, Dhody AN, Forrest S, et al. 2014. Second-pandemic strain of Vibrio cholerae from the Philadelphia cholera outbreak of 1849. N Engl J Med 370: $334-340$.

Doroghazi JR, Albright JC, Goering AW, Ju KS, Haines RR, Tchalukov KA, Labeda DP, Kelleher NL, Metcalf WW. 2014. A roadmap for natural product discovery based on large-scale genomics and metabolomics. Nat Chem Biol 10: 963-968.

Feng DF, Cho G, Doolittle RF. 1997. Determining divergence times with a protein clock: Update and reevaluation. Proc Natl Acad Sci 94: 13028-13033.

Forsberg KJ, Reyes A, Wang B, Selleck EM, Sommer MO, Dantas G. 2012. The shared antibiotic resistome of soil bacteria and human pathogens. Science 337: 1107-1111.
Forsberg KJ, Patel S, Gibson MK, Lauber CL, Knight R, Fierer N, Dantas G. 2014. Bacterial phylogeny structures soil resistomes across habitats. Nature 509: 612-616.

Gibson MK, Forsberg KJ, Dantas G. 2015. Improved annotation of antibiotic resistance determinants reveals microbial resistomes cluster by ecology. ISME J 9: 207-216.

Grishin NV. 1995. Estimation of the number of amino acid substitutions per site when the substitution rate varies among sites. J Mol Evol 41: 675-679.

Hall BG, Barlow M. 2003. Structure-based phylogenies of the serine $\beta$-lactamases. J Mol Evol 57: 255-260.

Hall BG, Barlow M. 2004. Evolution of the serine $\beta$-lactamases: Past, present and future. Drug Resist Updat 7: 111-123.

Hall BG, Salipante SJ, Barlow M. 2003. The metallo- $\beta$-lactamases fall into two distinct phylogenetic groups. J Mo Evol 57: 249-254.

Hall BG, Salipante SJ, Barlow M. 2004. Independent origins of subgroup $\mathrm{Bl}+\mathrm{B} 2$ and subgroup $\mathrm{B} 3$ metallo- $\beta$-lactamases. J Mol Evol 59: 133-141.

Humeniuk C, Arlet G, Gautier V, Grimont P, Labia R, Philippon A. 2002. $\beta$-lactamases of Kluyvera ascorbata, probable progenitors of some plasmid-encoded CTX-M types. Antimicrob Agents Chemother 46: 3045-3049.

Jansson JK, Tas N. 2014. The microbial ecology of permafrost. Nat Rev Microbiol 12: 414-425.

Knapp CW, Dolfing J, Ehlert PA, Graham DW. 2010. Evidence of increasing antibiotic resistance gene abundances in archived soils since 1940. Environ Sci Technol 44: $580-587$.

Landan G, Cohen G, Aharonowitz Y, Shuali Y, Graur D, Shiffman D. 1990. Evolution of isopenicillin N synthase genes may have involved horizontal gene transfer. $\mathrm{Mol}$ Biol Evol 7: 399-406.

Lynch M. 2010. Evolution of the mutation rate. Trends Genet 26: 345-352.

Mather AE, Baker KS, McGregor H, Coupland P, Mather PL, Deheer-Graham A, Parkhill J, Bracegirdle P, Russell JE, Thomson NR. 2014. Bacillary dysentery from World War 1 and NCTC1, the first bacterial isolate in the National Collection. Lancet 384: 1720.

Medema MH, Kottmann R, Yilmaz P, Cummings M, Biggins JB, Blin K, de Bruijn I, Chooi YH, Claesen J, Coates RC, et al. 2015. Minimum information about a biosynthetic gene cluster. Nat Chem Biol 11: 625-631.

Mindlin S, Minakhin L, Petrova M, Kholodii G, Minakhina S, Gorlenko Z, Nikiforov V. 2005. Present-day mercury resistance transposons are common in bacteria preserved in permafrost grounds since the upper pleistocene. Res Microbiol 156: 994-1004.

Moore AM, Patel S, Forsberg KJ, Wang B, Bentley G, Razia Y, Qin X, Tarr PI, Dantas G. 2013. Pediatric fecal microbiota harbor diverse and novel antibiotic resistance genes. PLoS ONE 8: e78822.

Ochman H, Lawrence JG, Groisman EA. 2000. Lateral gene transfer and the nature of bacterial innovation. Nature 405: 299-304.

Pehrsson EC, Forsberg KJ, Gibson MK, Ahmadi S, Dantas G. 2013. Novel resistance functions uncovered using functional metagenomic investigations of resistance reservoirs. Front Microbiol 4: 1-11. 
J. Perry et al.

Perron GG, Whyte L, Turnbaugh PJ, Goordial J, Hanage WP Dantas G, Desai MM. 2015. Functional characterization of bacteria isolated from ancient arctic soil exposes diverse resistance mechanisms to modern antibiotics. PLoS ONE 10: e0069533.

Petrova M, Gorlenko Z, Mindlin S. 2009. Molecular structure and translocation of a multiple antibiotic resistance region of a Psychrobacter psychrophilus permafrost strain. FEMS Microbiol Lett 296: 190-197.

Petrova M, Gorlenko Z, Mindlin S. 2011. Tn5045, a novel integron-containing antibiotic and chromate resistance transposon isolated from a permafrost bacterium. Res Microbiol 162: 337-345.

Petrova M, Kurakov A, Shcherbatova N, Mindlin S. 2014. Genetic structure and biological properties of the first ancient multiresistance plasmid pKLH80 isolated from a permafrost bacterium. Microbiology 160: 2253-2263.

Santiago-Rodriguez TM, Fornaciari G, Luciani S, Dowd SE, Toranzos GA, Marota I, Cano RJ. 2015. Gut microbiome of an 11 th century AD Pre-Columbian Andean mummy. PLOS ONE 10: e0138135.

Skippington E, Ragan MA. 2011. Lateral genetic transfer and the construction of genetic exchange communities. FEMS Microbiol Rev 35: 707-735.

Stokes HW, Gillings MR. 2011. Gene flow, mobile genetic elements and the recruitment of antibiotic resistance genes into Gram-negative pathogens. FEMS Microbiol Rev 35: 790-819.
Tatusov RL, Koonin EV, Lipman DJ. 1997. A genomic perspective on protein families. Science 278: 631-637.

van Elsas JD, Bailey MJ. 2002. The ecology of transfer of mobile genetic elements. FEMS Microbiol Ecol 42: 187197.

Wagner DM, Klunk J, Harbeck M, Devault A, Waglechner N, Sahl JW, Enk J, Birdsell DN, Kuch M, Lumibao C, et al. 2014. Yersinia pestis and the plague of Justinian 541-543 AD: A genomic analysis. Lancet Infect Dis 14: 319-326.

Wang C, Sui Z, Leclercq SO, Zhang G, Zhao M, Chen W, Feng J. 2015. Functional characterization and phylogenetic analysis of acquired and intrinsic macrolide phosphotransferases in the Bacillus cereus group. Environ Microbiol 17: 1560-1573.

Warinner C, Rodrigues JF, Vyas R, Trachsel C, Shved N, Grossmann J, Radini A, Hancock Y, Tito RY, Fiddyment S, et al. 2014. Pathogens and host immunity in the ancient human oral cavity. Nat Genet 46: 336-344.

Weigel BJ, Burgett SG, Chen VJ, Skatrud PL, Frolik CA, Queener SW, Ingolia TD. 1988. Cloning and expression in Escherichia coli of isopenicillin $\mathrm{N}$ synthetase genes from Streptomyces lipmanii and Aspergillus nidulans. J Bacteriol 170: 3817-3826.

Wiedenbeck J, Cohan FM. 2011. Origins of bacterial diversity through horizontal genetic transfer and adaptation to new ecological niches. FEMS Microbiol Rev 35: 957-976.

Wright GD. 2010. Antibiotic resistance in the environment: A link to the clinic? Curr Opin Microbiol 13: 589-594. 


\section{$\&_{\mathrm{CSH}}^{\infty} \&$ Cold Spring Harbor

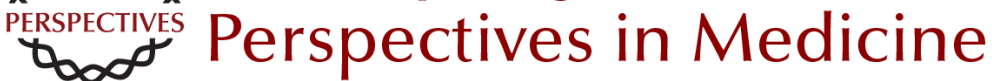

\section{The Prehistory of Antibiotic Resistance}

Julie Perry, Nicholas Waglechner and Gerard Wright

Cold Spring Harb Perspect Med 2016; doi: 10.1101/cshperspect.a025197

Subject Collection Antibiotics and Antibiotic Resistance

Fosfomycin: Mechanism and Resistance Lynn L. Silver

Pleuromutilins: Potent Drugs for Resistant Bugs

--Mode of Action and Resistance Susanne Paukner and Rosemarie Riedl

Appropriate Targets for Antibacterial Drugs Lynn L. Silver

Lincosamides, Streptogramins, Phenicols, and Pleuromutilins: Mode of Action and Mechanisms of Resistance

Stefan Schwarz, Jianzhong Shen, Kristina Kadlec, et al.

Resistance to Macrolide Antibiotics in Public Health Pathogens

Corey Fyfe, Trudy H. Grossman, Kathy Kerstein, et al.

Bacterial Protein Synthesis as a Target for

Antibiotic Inhibition

Stefan Arenz and Daniel N. Wilson

Antibacterial Antifolates: From Development

through Resistance to the Next Generation

Alexavier Estrada, Dennis L. Wright and Amy C. Anderson

Antibacterial Drug Discovery Targeting the Lipopolysaccharide Biosynthetic Enzyme LpxC Alice L. Erwin
The Whys and Wherefores of Antibiotic

Resistance

Cameron R. Strachan and Julian Davies

$\beta$-Lactamases: A Focus on Current Challenges Robert A. Bonomo

Approved Glycopeptide Antibacterial Drugs: Mechanism of Action and Resistance Daina Zeng, Dmitri Debabov, Theresa L. Hartsell, et al.

Mechanism of Action and Resistance to Daptomycin in Staphylococcus aureus and Enterococci William R. Miller, Arnold S. Bayer and Cesar A. Arias

Polymyxin: Alternative Mechanisms of Action and Resistance

Michael J. Trimble, Patrik Mlynárcik, Milan Kolár, et al.

Topoisomerase Inhibitors: Fluoroquinolone

Mechanisms of Action and Resistance

David C. Hooper and George A. Jacoby

$\beta$-Lactams and $\beta$-Lactamase Inhibitors: An

Overview

Karen Bush and Patricia A. Bradford

Rifamycins, Alone and in Combination David M. Rothstein

For additional articles in this collection, see http://perspectivesinmedicine.cshlp.org/cgi/collection/ 